\title{
Three Online Neutron Beam Experiments Based on the iLab Shared Architecture
}

\author{
doi:10.3991/ijoe.v7i1.1484 \\ Kimberly DeLong, V. Judson Harward, Philip Bailey, \\ James Hardison, Gordon Kohse and and Yakov Ostrocsky \\ Massachusetts Institute of Technology, Cambridge, Massachusetts, USA
}

\begin{abstract}
Students at MIT have traditionally executed certain experiments in the containment building of the MIT nuclear reactor as part of courses in Nuclear Engineering and the third year laboratory course for Physics majors. A joint team of faculty and research staff from the MIT Nuclear Reactor Laboratory (MIT-NRL) and MIT's Center for Educational Computing Initiatives have implemented online versions of three classic experiments; (a) a determination of MIT reactor coolant temperature through measurement of thermal neutron velocity, (b) a demonstration of the DeBroglie relationship of the kinetic energy and momentum of thermal neutrons and study of Bragg diffraction through a single copper crystal at various orientations, and (c) a measurement of beam depletion using a variety of shielding filters. These online experiments were implemented using the LabVIEW® virtual instrumentation package and the interactive version of the iLab Shared Architecture (ISA). Initial assessment of the online experiments indicates that they achieve comparable educational outcomes to traditional versions of the labs executed in the reactor containment building.
\end{abstract}

Index Terms - remote Labs, online labs, iLab architecture, web services, engineering education, nuclear physics.

\section{BACKGROUND}

Online laboratories (iLabs) are experimental facilities that can be accessed through the Internet, allowing students and researchers to carry out experiments from anywhere at any time. They harness the Internet and enable students to access and utilize real instruments remotely. These types of remote labs enrich science and engineering education by vastly increasing the scope and variety of experiments that students are able to access. Experiments allow a student to compare reality with simulations, collaborate with each other, and follow their curiosity. Conducting experiments motivates students and causes them to learn more effectively.

However, many science and engineering classes do not include a traditional lab component due to the prohibitive expense involved in staffing and operating labs, space and logistical requirements and safety considerations. By providing online access to remote laboratories, iLabs are delivering the educational benefits of hands-on experimentation over the Internet to our own students and to students around the world.

Remote laboratories allow for the more efficient use of laboratory equipment and can give students access to exciting and unique resources. In this case, the Spectrometer iLab project's goal is to provide educational opportunities to students at various educational levels nationwide and internationally that do not have the benefit of an on-site nuclear reactor or other neutron source.

\section{The ILAB SHARED ARChITECTURE}

The iLab Project at MIT has focused on the design of a common architecture for the development and deployment of online laboratories called the iLab Shared Architecture (ISA) [1]. ISA is a robust, scalable, open-source infrastructure built on web services that provide a unifying software framework to support access to a wide variety of online laboratories. The ISA provides a set of generic lab services, such as user account management, scheduling and data storage, in a middleware system that can be accessed using web services. Students and the online laboratories can be globally distributed across an arbitrary number of locations linked only by the Internet. Users access these remote laboratories through single sign-on and a simple standard administrative interface.

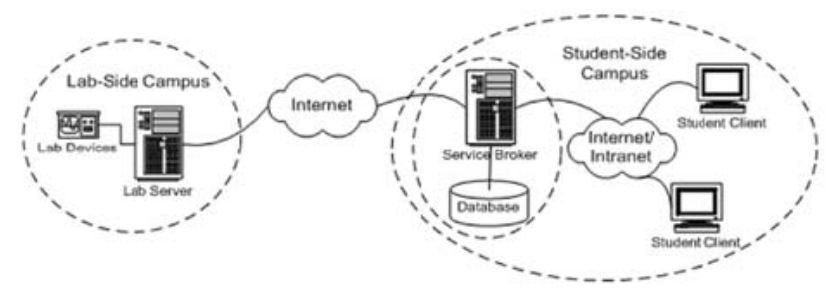

Figure 1. ISA components: Service Broker, Lab Server and Lab Client

A founding principle on which iLabs is built is the separation of responsibility. iLabs separate the responsibility for lab development from that of managing information about the students using the lab. Not only do the services provided by the iLab Service Broker (authentication, authorization, experiment storage, user management) simplify the task of implementing a particular experiment, but the location of the iLab services on a separate server divorces management of students on the Service Broker from management of the lab experiment on the lab server. In a typical configuration, as shown in Figure 1, a campus using iLab-based experiments will run a Service Broker administered by IT staff responsive to their own faculty and students. This Service Broker can access multiple lab servers potentially located at multiple remote campuses anywhere on the Internet. The students' accounts and their experiment storage reside on their local campus Service Broker regardless of where the experiment itself is executed. The lab server team need not be aware of which student is using their equipment at any given time, but they are 
assured that the student comes from an approved campus. The separation of responsibilities gives iLabs unique scaling properties and potentially enormous economic advantages with revolutionary impact in science and engineering education at all levels.

The iLab Shared Architecture is divided into three major components; Service Broker and related service, Lab Server and Lab Client [2].

The Service Broker serves as the heart of the ISA. It provides generic administrative services, such as authentication, authorization, user management, scheduling and data storage. The design of the Service Broker strictly separates the task of publishing an iLab from that of managing the students using it. This separation encourages the sharing of such experiments between institutions. The Service Broker also serves as the gateway in inter-institutional relationships. Once two institutions have mutually registered their Service Brokers with each other it becomes much easier to share iLabs.

The Lab Server is the component connected directly to the lab equipment and deals with the actual operation of the experiment hardware. It is the administrative interface to the lab equipment and enables labratory administrators to setup and configure each experiment independently. Each lab server is specific to its experiment hardware, and as such, a different lab server must be used for each set of lab instrumentation.

The generic components of the iLab Shared Architecture require communication to be via web services. But the architecture leaves decisions regarding the construction of the experiment to the domain expert and lab developer. This allows developers to use custom, even proprietary, technology both for constructing their lab client and lab server and for managing the experiment based communication between them. Not only does this provide support for specific, potentially high-bandwidth lab experiments, but it also enables support for preexisting lab control software. In the case of the Spectrometer iLab, we use the National Instruments LabVIEW ${ }^{\circledR}$ platform for both the lab hardware control and the lab client interface.

The Spectrometer experiment utilizes the MIT iLab project's open source reference implementation of the Lab Server called the LabVIEW® Integrated Interactive Lab Server (LVILS). The LVILS provides a standard way for interactive lab developers to interface the generic ISA services to LabVIEW ${ }^{\circledR}$ instrument control software. In the LVILS, as in the interactive model generally, the lab client is developed in close relation with the lab server and corresponding instrumentation. The LVILS furnishes support for accessing the ISA services such as experiment storage and scheduling, as well as interfacing to LabVIEW ${ }^{\circledR}$ Virtual Instruments.

The Lab Client is the interface through which students access the iLab. It provides an intuitive representation of the iLab being run, allowing users to specify parameters and interact with the lab hardware. The ISA supports many client technologies including Java applets, Java Server pages, Windows Forms clients, and LabVIEW® remote front panels.

The ISA supports multiple Lab Clients that utilize the same backend Lab Server and experiment hardware. Building educationally valuable online labs is expensive and it is necessary to leverage, where ever possible, this investment by encouraging the sharing of lab resources. One way to share labs is to develop new educationally appropriate interfaces targeted to specific audiences. The ISA offers a scalable solution to improving STEM education by growing the use of online labs to increase inquiry-based learning and encouraging student interest in science through experimentation. We hope to bridge the traditional gap between the university, secondary school and other communities while recognizing differences in educational context and goals.

Consider, for example, the manipulations required to perform a basic time-of-flight (TOF) spectroscopy experiment. With the chopper wheel motor running, the low efficiency BF3 detector is positioned close to the chopper wheel and data is accumulated using a multichannel scaler (MSC) card. The detector must then be repositioned much further from the chopper and the data acquisition process repeated. For this experiment, we plan to develop at least three different interfaces each targeted at a different educational audience.

We envision a variety of client implementations to accommodate the needs of different educational audiences. For high school students, we need a simpler client that is focused on a single experiment which includes a set of predefined detector positions and some initial setting of the counting parameters. This will allow high school students to step through the experiment and observe accumulation of real data with little possibility of experimental errors that might interfere with basic data analysis and understanding.

For advanced undergraduate or graduate students, there is our current client, where students have access to all three experiments and they select the experiment at run time. In this interface users are allowed to determine detector positions for themselves, check and adjust alignment of the detector with the beam and set various MCS parameters.

One could also imagine additional hardware being added to the experiment implementation for research purposes and new clients being developed specifically for research staff. Each of the clients described would use some if not all of the hardware features currently implemented in the Spectrometer iLab but would be geared towards a specific group or classes need.

The iLab team is currently developing a simpler version of the beam depletion or neutron absorption experiment for use in advanced secondary school courses in cooperation with the University of Queensland, Australia and the Queensland Academy for Science, Math, and Technology (QUASMT). We plan to begin beta testing next summer.

\section{THE SPECTROMETER EXPERIMENTS}

In the early 1980s, Nobel Prize winning physicist Clifford Shull collaborated with the MIT Nuclear Reactor Laboratory (MIT-NRL) to build the neutron spectrometer facility so that MIT undergraduates could replicate basic neutron science and physics experiments. These experiments utilize a beam port which provides a continuous beam of well-thermalized neutrons from the heavy water reflector region of MIT's 5MW research reactor. The experiments are part of courses in Nuclear Engineering and the third year laboratory course for Physics majors [3]. The experiments developed include: 
- Measurement of the Maxwell Boltzmann Distribution of Thermal Neutrons. The objective of this experiment (also called the Time-of-Flight Neutron Physics experiment) is to measure the energy spectrum of the beam neutrons using time-offlight techniques, and then to compare the results with the expected Maxwell Boltzmann distribution that is predicted. Students are asked to perform measurements at two points in the beam guide tube using neutron detectors, multichannel scalers, and oscilloscopes. Students plot the data they gather and attempt to calculate thermal neutron velocity and flux density.

- Demonstration of the DeBroglie relationship of the kinetic energy and momentum of thermal neutrons and demonstration of Bragg Diffraction. The objective of this experiment is to demonstrate neutron diffraction using a copper single crystal. Students are asked to measure the Bragg-diffracted beam that results when the neutron beam interacts with the crystal. They perform several measurements at various angles of incidence, and are asked to plot their data to demonstrate the DeBroglie relation.

- Demonstration of Beam Depletion or Shielding Effectiveness in a Neutron Beam. The objective of this experiment is to demonstrate thermal neutron behavior in the presence of various absorption materials. After characterizing the "open" beam in the TOF experiment, the students place samples of materials such as lead, cadmium, water and borated polyethylene in the beam and repeat their energy spectrum measurement. Based on the data collected, students are asked to calculate cross-section or attenuation coefficient of the materials and qualitatively evaluate how these parameters depend on neutron energy.

The neutron spectrometer was initially designed as a nonautomated facility installed in the Massachusetts Institute of Technology Research Reactor (MITR) which utilized the 4DH1 beam port. Students entering the reactor containment building to utilize one of the experiments are required to complete General Employee Radiological Training (GERT) and become radiation workers by federal law. These requirements and other security issues have limited the number of students able to utilize the spectrometer experiments. In 2007, with funding from the Department of Energy's Innovations in Nuclear Infrastructure Education grant for the New England Consortium and funds from the MIT iCampus project, a joint team of faculty and research staff from the MIT Nuclear Reactor Laboratory (MITNRL) [4] and MIT's Center for Educational Computing Initiatives [5] was created to enable online access and usage of these experiments based on the iLab Shared Architecture. Figure 2 shows a rendering of the principal components involved with these experiments. They include a mechanical chopper, a low efficiency detector, a crystal, a high efficiency detector and a materials attenuation slide.

\section{THE IMPLEMENTATION}

The student spectrometer hardware implementation can be described in terms of three major subsystems. The first of these consists of the equipment required to manipulate and detect a low energy neutron beam from the MITR re-

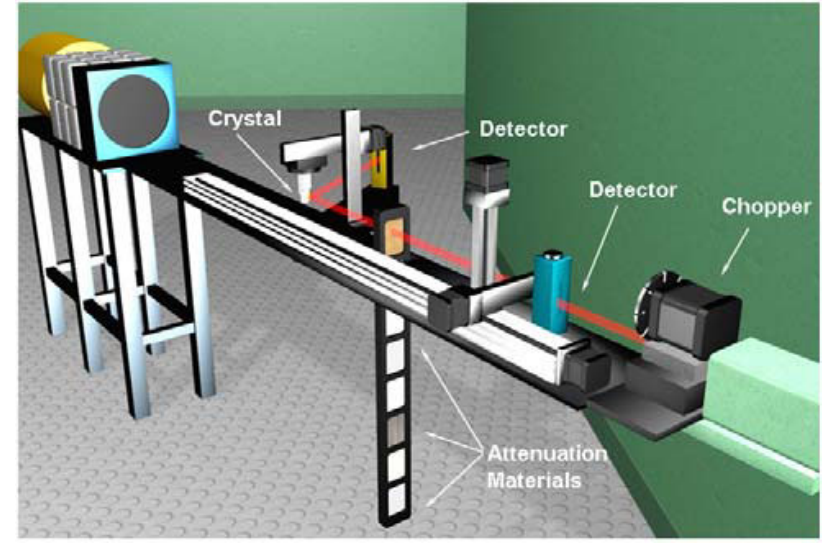

Figure 2. Rendering of the principal components of the spectrometer experiments by R. Mark Bessette (MIT).

search reactor and is essentially unchanged from the established version of the experiment that has been run locally by MIT undergraduate and graduate students for several decades. The main components are a collimator and chopper wheel that produce a narrow, pulsed, beam of neutrons, a low-efficiency BF3 detector (LND Model 201) that is used to count the neutrons in the direct beam, a copper single crystal to diffract the neutrons and a high efficiency $3 \mathrm{He}$ detector (LND Model 251) that is used to count the neutrons in the diffracted beam. The detectors are powered by a dual high voltage power supply (Canberra Model 3125) and the signal from each detector is processed by a preamplifier and amplifier/timing single channel analyzer (TSCA) (Canberra Model 2006 and Ortec 590, respectively). These components are mounted in a standard nuclear instrumentation module (NIM) bin. The TSCA produces a sharp voltage pulse corresponding to each detected neutron that is processed by a multichannel scaler (MCS) board (Ortec MCS-pciтM) installed in the local PC. The MCS is gated by a signal produced by a light sensor that detects the passing chopper wheel slits. Accumulating data in the MCS produces a time of flight (TOF) spectrum of the neutrons in the beam that can be converted to an energy spectrum for analysis of the neutron energy distribution.

The second subsystem consists of components used to position the detectors and other elements of the experimental apparatus such as the single crystal and a set of materials that can be positioned in the beam to measure their neutron attenuation properties. Most of the detector motions are accomplished using stepper motors (Automation Direct, various models) with linear slides or rotary tables (Velmex, Inc., various models).

The low efficiency detector has three linear axes of motion: two orthogonal axes perpendicular to the beam and one axis in the beam direction to allow spectra to be generated at varying distances from the chopper wheel. The single crystal has two linear axes of motion for positioning in the beam and one rotational axis to align the crystal planes at varying angles to the neutron beam. The high efficiency detector has one axis of motion driven by a linear slide but producing a rotation centered on the crystal position in the beam. This motion is used to find diffracted neutron beams. A stepper-motor-driven rotary table is also used to select different materials for attenuation experiments. In addition to the stepper motor systems, there are relay-controlled pneumatic actuators to 


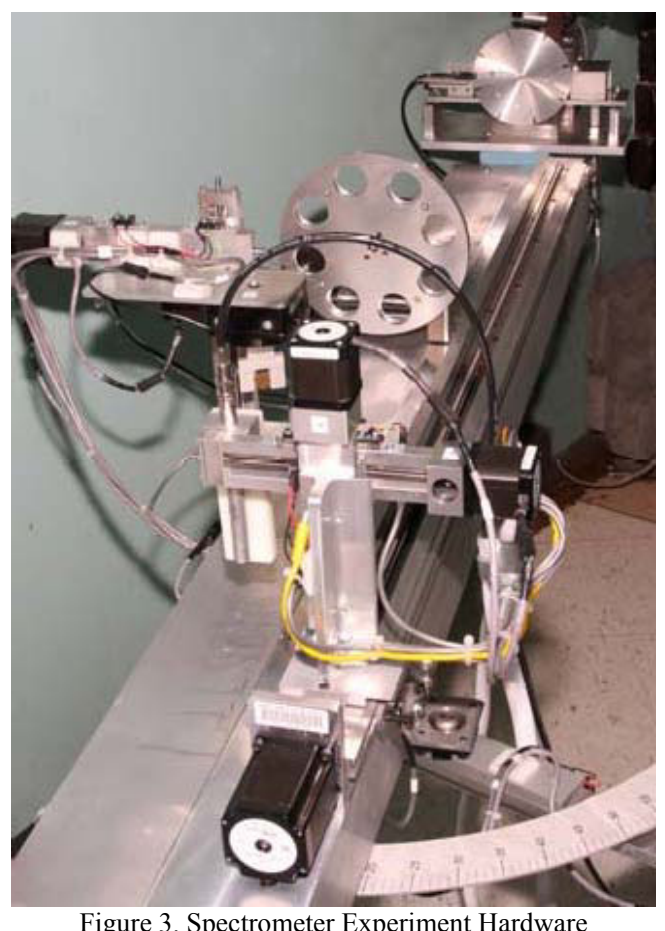

move the attenuation material setup in and out of the beam and to raise and lower the chopper wheel to allow a "white" (unchopped) beam to be produced. A relay is also provided to start and stop the chopper wheel motor. Figure 3 shows the finished experiment implementation of the main components.

The third subsystem is a National Instruments PXIbased data acquisition and control system that provides the interface between the first two subsystems and the LabVIEW ${ }^{\text {TM }}$ Virtual Instrument (VI) client that is used to perform the experiment in either local or remote (iLabs) mode. Components are housed in a PXI-1042Q chassis communicating with the local PC over a PXI-8360 interface card. There are 3 PXI-7330 motion control boards operating the stepper motors and associated limit switches, a PXI-6259 multi-function DAQ to receive signals from additional limit switches on the pneumatically controlled motions, a PXI-6521 8 channel relay card to drive the relays described above and a PXI6602 timing I/O board to acquire time-independent counting data from the detectors (i.e. independent of the MCS system).

The Spectrometer client interface, called a Virtual Instrument or VI, was also implemented using LabVIEW® from National Instruments. LabVIEW is a graphical, eventdriven programming package optimized for data acquisition, instrument control, and the graphical display of data. For this experiment, students need to download and install the LabVIEW ${ }^{\circledR}$ plugin for their browser.

Figure 4 shows the Spectrometer LabVIEW ${ }^{\circledR}$ client for the Maxwell Boltzmann Distribution Experiment otherwise known as the Time-of-Flight neutron physics experiment. In the client, students use the "Motion Control" area to position the Low Efficiency detector, control the choppers position and spin, and set parameters in the MultiChannelScaler (MCS) tab for the experiment. While the experiment is underway, students can watch the

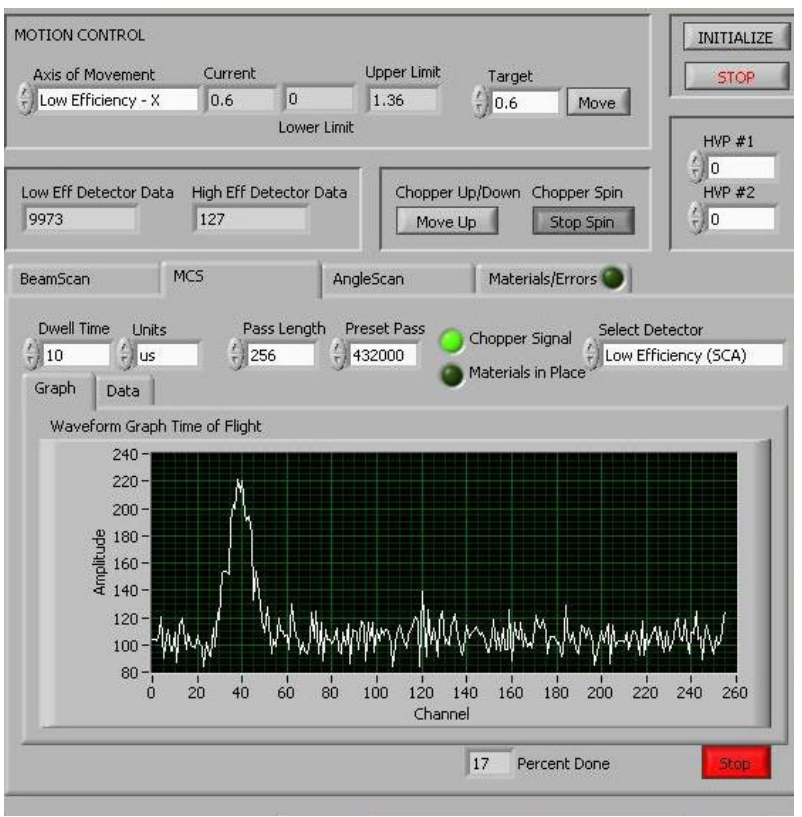

Figure 4. LabVIEW® client for the Maxwell Boltzmann Distribution Experiment otherwise known as the Time-of-Flight Neutron PhysicsExperiment.

counts both by numbers counted in the Low Eff Detector Data field and graphically in the Waveform Graph of the Time of Flight data.

\section{RUNNING AN EXPERIMENT}

The Spectrometer iLab is an interactive experiment that requires users to schedule experiment time in advance since typical experiment times can range from 20 minutes to 8 hours. Scheduling in advance ensures that the operators in the reactor control room can open the beam port. The beam port is only opened when an experiment is scheduled. Students typically work in teams of two or three.

To run an experiment, students login to the Service Broker, select the Spectrometer experiment group and redeem the reservation they scheduled in advance. The Service Broker checks to make sure that the user has a valid reservation and that he/she is authorized to use the Spectrometer experiment. The user is then presented with a "Launch Lab" button and is able to start the experiment client, as shown in Fig. 5. When the experiment is launched, the Service Broker facilitates the exchange of credentials so that the Lab Client running in the student's browser can talk directly with the Lab Server and experiment hardware. The LabVIEW ${ }^{\mathrm{TM}}$ client VI is launched from the LVILS Lab Server and displayed in the user's browser using a LabVIEW ${ }^{\mathrm{TM}}$ plugin. The client also establishes communications with the datasocket server so that experiment data can be stored in the database for later retrieval.

The Spectrometer experiment installation has two web Cameras available so users can watch the experiment while in progress. We have found that the cameras are essential to the student understanding and believing that they are working on and controlling real hardware. The first camera, Fig 6, is an overall view of the experiment and allows the user to watch the detectors and crystal as they are being moved. The second camera, Fig 6 , is a 


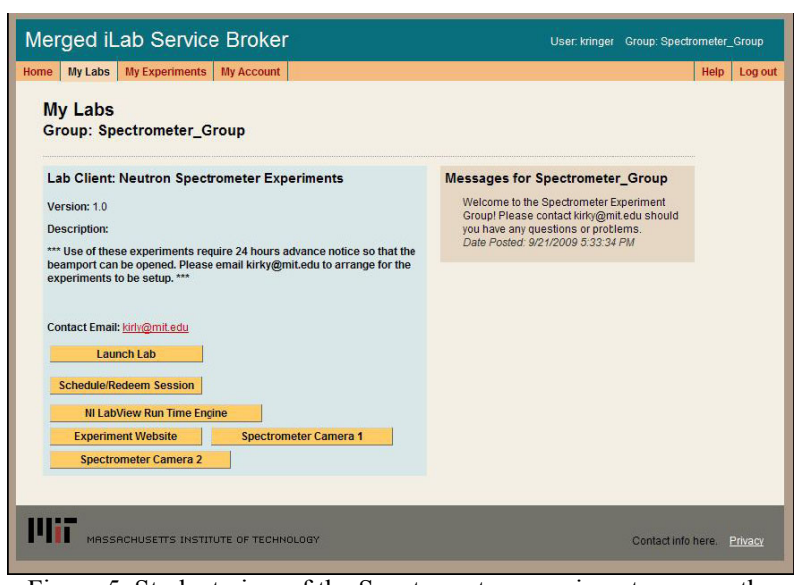

Figure 5. Student view of the Spectrometer experiment page on the Service Broker.

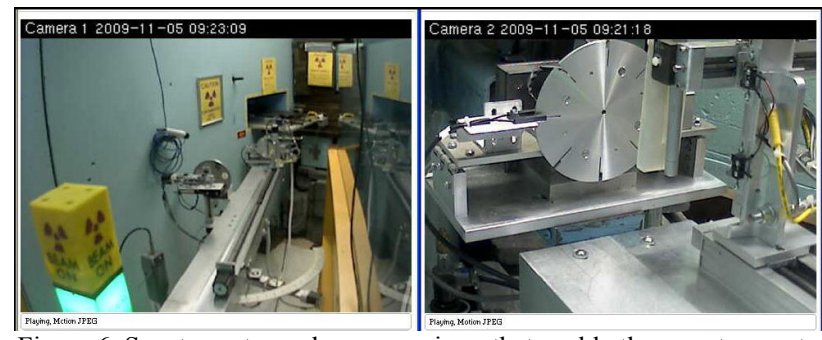

Figure 6. Spectrometer web camera views that enable the remote user to view the experiment while in progress.

close-up view of the chopper wheel. We have developed animations of each of the experiments to give the user a visual understanding of the phenomena being explored and the experiment being performed. Figure 7. Animation stills from the Spectrometer beam depletion experiment showing Aluminum and Polyethelene by R. Mark Bessette (MIT).

In this version of the Spectrometer client, shown in figure 4 , the user can run any of the three experiments by selecting the correct tab. Before beginning any experiment, there are several steps that all users should take. First, the experiment hardware must be initialized. This will run an initialization sequence to make sure that all the components are in their correct starting positions. Second, students use the BeamScan tab to find the centerline of the beam, by moving the low efficiency detector across the beam port area. Users will see a peak in counts when the Low Efficiency Detector is centered in the beam. Once the user knows where the center of the beam is, they can position any of the components in the correct location.

In the time-of-flight (TOF) experiment, students use the MCS tab to perform measurements at two points (near and far) in the beam using neutron Low Efficiency Detectors and multichannel scalers. Utilizing the AngleScan tab, students can control the copper crystal's position and rotation as well as the high-efficiency detector angular position. In the Materials/Error tab, users can control the sample carousel and position various absorption materials into the beam for testing. A combination of hardware and software safety interlocks is used to avoid collisions of the movable components and to establish usable ranges for each experiment component.
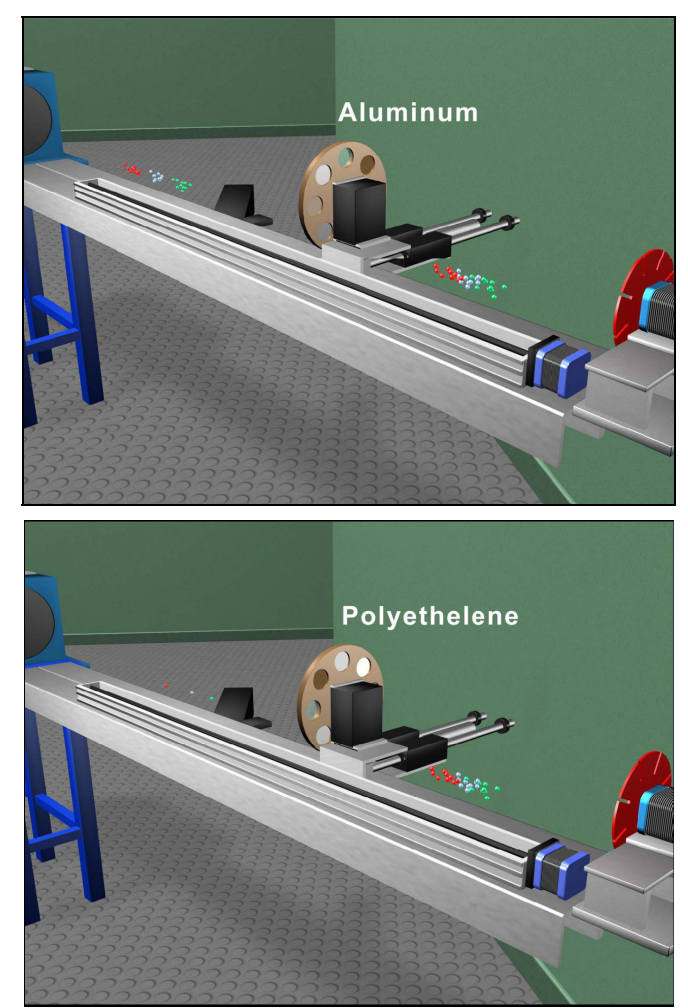

Figure 7. Animation stills from the Spectrometer beam depletion experiment showing Aluminum and Polyethelene by R. Mark Bessette (MIT).

\section{USAGE}

Most of the usage to date has been by undergraduate students at MIT in the departments of Nuclear Science and Engineering (NSE) and Physics. These students generally have at least one "local" session at the experiment site before being offered the option to continue their work online.

Approximately half the NSE students doing the lab as part of a required course used the on-line option, while the fraction was much higher for the Physics students. We attribute this largely to the fact that the NSE students do not perform the diffraction portion of the lab and thus require much less counting time than the Physics students. One of the clear benefits of online access is that the students accumulate counting data over much longer times and obtain better quality data sets that are more amenable to analysis. Students are also more likely to repeat experiments if data anomalies are discovered.

\section{SCHEDULING}

Currently due to regulations and security issues and to avoid unnecessary activation of the beam components, the beam port is not open 24/7. The beam port is opened and closed manually by the operator on duty inside the reactor containment facility only when an experiment is scheduled. We currently add the scheduled experiments to the operator's schedule manually, that is to say, the Service Broker manager periodically looks at the scheduling calendar and tries to send an email at least 24 hours in advance to the reactor operator manager with a list of scheduled experiments and request that the beam port be opened. The Reactor operations manager then adds the beam port open and closes to the operator's schedule. 
This process has several possible points where errors can occur. First, we request that students schedule their usage more than 24 hours in advance for weekday and no later than Friday midday for the weekend. We try very hard to work with the students to ensure they have access to the experiment, but this is sometimes an issue when a student makes a reservation through the Service Broker at 4:55pm on Friday afternoon for time on Sunday. There is no guarantee that the Service Broker manager will see the new reservation or that it can be added to the operator's weekend schedule. Second, the list of scheduled experiments is collated from the Service Broker by hand and sent via email. Sometimes this involves two or three email exchanges a day and sometimes emails are missed. And lastly, manual transmissions of schedules that pass daily through human hands are prone to transcription, typing or misreading errors. While we cannot solve the regulatory and security issues, we hope to improve and automate part of the scheduling process in a future release of the Service Broker. We will be adding direct email notification to a mailing list (which will include the reactor operators) when an experiment is scheduled or changed. In addition, we hope to improve scheduling features to make it more user friendly and enable faculty and students to see who has the reserved time in case schedules need to be negotiated. Right now, reservations are on a first come basis.

\section{FUTURE WORK}

A variety of hardware upgrades are being planned or considered. A larger, more robust detector Z-axis motion slide with a higher power stepper motor would improve reliability of the system and reduce the required frequency of mechanical maintenance. The multi-channel scaler is fully controllable from within LabView ${ }^{\mathrm{TM}}$ and thus within the iLabs interface, but the "front end" of the nuclear counting system consisting of the high voltage power supplies and amplifier/TSCAs can neither be controlled remotely nor report their parameter settings to a computer interface. As new types of computerbased nuclear instrumentation become available, an appropriate replacement for the NIM electronics may be identified to provide this capability.

Some software improvements are also planned. Minor changes to the LabView ${ }^{\mathrm{TM}}$ interface will be made to incorporate suggestions from students and developers for improving appearance and easing navigation and operation. More substantive changes include the addition of counter/scaler and oscilloscope functionality to the interface. The counter/scaler would emulate the traditional hardware version with controls for counting time, single or repeat counts and automatic or manual count reset. This is a relatively minor programming effort because most of the functionality already exists in the Beam Scan and Angle Scan modules. An oscilloscope function that would allow on-line users to view the output pulses of the detector pre-amplifiers and amplifiers would be a significant enhancement to the on-line implementation and would be a preparatory step for putting the nuclear instrumentation under LabView ${ }^{\mathrm{TM}}$ control as discussed above. Some of the hardware required is already in place but additions would probably be necessary to allow switching the oscilloscope input between the lowefficiency and high efficiency detectors.

\section{ACKNOWLEDGMENT}

The authors wish to thank our colleagues, Judy Maro, David Litster, Raymond Ma, Lin-wen Hu, Timothy Lucas, Meg Westlund and Maria Karatzas, whose vision, hard work and insights has guided successfully the development and deployment of the Spectrometer iLab.

A version of this paper was presented in the IEEE EDUCON 2010 conference and selected for submission to this journal. It received a co-award in the Infrastructure and Technologies for Engineering Education area of this conference.

\section{REFERENCES}

[1] Harward, V.J., et al, "The iLab Shared Architecture: A Web Services Infrastructure to Build Communities of Internet Accessible Laboratories," Proceedings of the IEEE, vol.96, no.6, pp.931-950, June 2008. doi:10.1109/JPROC.2008.921607

[2] Hardison, J., DeLong, K., Bailey, P., Harward, V.J., "Deploying Interactive Remote Labs Using the iLab Shared Architecture," Frontiers in Education (FIE) Conference, Saratoga Springs, New York, October 22-25, 2008. iLab

[3] MIT Neutron Physics experiment description: http://web.mit.edu/8.13/www/38.shtml.

[4] Kohse, G., et al, "Progress Toward Deployment of a WebEnabled Neutron Spectrometer," Transactions of the American Nuclear Society, Vol. 96, No. 1, pp. 403-404, June 2007.

[5] Wiki: http://ilab.mit.edu/wiki.

\section{AUTHORS}

Kimberly DeLong is with the Center for Educational Computing Initiatives, Massachusetts Institute of Technology, Cambridge, Massachusetts 02139 USA USA (e-mail: kirky@mit.edu).

V. Judson Harward is with the Center for Educational Computing Initiatives, Massachusetts Institute of Technology, Cambridge, Massachusetts 02139 USA USA (e-mail: jud@mit.edu).

Philip Bailey is with the Center for Educational Computing Initiatives, Massachusetts Institute of Technology, Cambridge, Massachusetts 02139 USA USA (e-mail: pbailey@mit.edu).

James Hardison is with the Center for Educational Computing Initiatives, Massachusetts Institute of Technology, Cambridge, Massachusetts 02139 USA USA (e-mail: hardison@mit.edu).

Gordon Kohse is with the Nuclear Reactor Laboratory, Massachusetts Institute of Technology, Cambridge, Massachusetts 02139 USA USA (e-mail: kohse@mit.edu).

Yakov Ostrocsky is with the Nuclear Reactor Laboratory, Massachusetts Institute of Technology, Cambridge, Massachusetts 02139 USA USA (e-mail: yakov@mit.edu).

This work was supported in part by the National Science Foundation under grant OCI-0753324. However, any opinions, findings, conclusions, and/or recommendations are those of the investigators and do not necessarily reflect the views of the Foundation.

Manuscript received 22 October 2010. Published as resubmitted by the authors 20 January 2011. 